\title{
A NOTE ON THE EQUATION $x^{2}=y^{q}+1$
}

\author{
E. Z. CHEIN
}

ABSTRACT. It is proved here that the equation $x^{2}=y^{q}+1$ has no solution in natural numbers $x, y$ for which $q$ is a prime $>3$.

It was shown by Chao Ko [1], [2] that the equation $x^{2}=y^{q}+1$ has no solution in natural numbers $x, y$ where $q$ is a prime $>3$.

It is the purpose of this note to give a simpler proof of Ko Chao's result.

Throughout this note all symbols denote natural numbers, and notation $(a, b)=g$ means $g$ is the greatest common divisor of $a$ and $b$.

Auxiliary lemmas. Lemmas 1 and 2 are collections of some well-known results. The third is due to Nagell [3].

Lemma 1. If $q$ is an odd prime and $(x, y)=1$, then $x+y$ divides $x^{q}+y^{q}$ and $\left(x+y,\left(x^{q}+y^{q}\right) /(x+y)\right)=q$ or 1 according as $x+y$ is divisible by $q$ or not.

Lemma 2. All the primitive solutions of equation $x^{2}+y^{2}=z^{2}$ for which $y$ is an even number are given by the formulas

$$
x=a^{2}-b^{2}, \quad y=2 a b \quad \text { and } \quad z=a^{2}+b^{2} \quad(a>b) .
$$

LemMA 3. If $x^{2}=y^{q}+1$ with $q$ prime and $x \geqslant 1, y \geqslant 1$, then $2 \mid y$ and $q \mid x$.

\section{Principal result.}

THEOREM. Let $q$ be a prime $>3$. The equation $x^{2}=y^{q}+1$ has no solution in natural numbers.

Proof. Let us assume now that there exist $x, y$ and a prime $q$ for which $x^{2}=y^{q}+1$.

It follows from Lemma 3 that $q \mid x$ and $2 \mid y$. Since $2 \nmid x$, by Lemma 3 we have $(x+1, x-1)=2$. Thus either

(I) $x+1=2^{q-1} y_{1}^{q}, \quad x-1=2 y_{2}^{q}$, or

(II) $x+1=2 y_{2}^{q}, \quad x-1=2^{q-1} y_{1}^{q}$

holds, where $y=2 y_{1} y_{2}, 2 \nmid y_{2}$ and $\left(y_{1}, y_{2}\right)=1$.

Case I. Suppose $x+1=2^{q-1} y_{1}^{q}$ and $x-1=2 y_{2}^{q}$. It follows from $y_{2}^{q}$ $=2^{q-2} y_{1}^{q}-1$ that

$$
\left(y_{2}^{2}\right)^{q}+\left(2 y_{1}\right)^{q}=\left(y_{2}^{q}+2\right)^{2}=((x+3) / 2)^{2} .
$$

Since $q \mid x$ and $q>3$ we see $q \nmid(x+3) / 2$; thus

Received by the editors June 3, 1975.

AMS (MOS) subject classifications (1970). Primary 10B15. 


$$
\left(y_{2}^{2}+2 y_{1},\left(\left(y_{2}^{2}\right)^{q}+\left(2 y_{1}\right)^{q}\right) /\left(y_{2}^{2}+2 y_{1}\right)\right)=1,
$$

by Lemma 3.

In view of (1), it follows that $y_{2}^{2}+2 y_{1}=h^{2}$ where $h \mid(x+3) / 2$. This gives

$$
\left(h y_{2}\right)^{2}+y_{1}^{2}=\left(y_{2}^{2}+y_{1}\right)^{2} \text {. }
$$

Since $\left(y_{1}, y_{2}\right)=1$, this implies $\left(h y_{2}, y_{1}\right)=1$. We observe that since $y_{2}$ is odd, so is $h$; then $4 \mid h^{2}-y_{2}^{2}$ so that $2 \mid y_{1}$.

By Lemma 2, the solutions of (2) are given by

$$
h y_{2}=a^{2}-b^{2}, \quad y_{1}=2 a b \quad \text { and } y_{2}^{2}+y_{1}=a^{2}+b^{2} \quad(a>b) .
$$

Therefore, $(a-b)^{2}=\left(y_{2}^{2}+y_{1}\right)-y_{1}=y_{2}^{2}$ which implies $y_{2}=a-b$, and so $y_{1}-y_{2}=2 a b-(a-b)=a(2 b-1)+b>0$, hence $y_{1}>y_{2}$. However, $y_{2}^{q}$ $=2^{q-2} y_{1}^{q}-1>y_{1}^{q}$ implies $y_{2}>y_{1}$, and this is impossible. This completes Case I.

Case II. The proof for this case proceeds similarly. It can be easily seen from $\left(y_{2}^{2}\right)^{q}-\left(2 y_{1}\right)^{q}=\left(y_{2}^{q}-2\right)^{2}=((x-3) / 2)^{2}$ follows $y_{2}^{2}-2 y_{1}=h^{2}$ where $h \mid(x-3) / 2$; this implies $\left(h y_{2}\right)^{2}+y_{1}^{2}=\left(y_{2}^{2}-y_{1}\right)^{2}$ so that Lemma 2 gives

$$
h y_{2}^{2}=a^{2}-b^{2}, \quad y_{1}=2 a b, \quad y_{2}^{2}-y_{1}=a^{2}+b^{2} \quad(a>b) .
$$

Hence $y_{1}-y_{2}=2 a b-(a+b)=(a-1)(b-1)+(a b-1)>0$ which is impossible because of $y_{2}^{q}=2^{q-2} y_{1}^{q}+1>y_{1}^{q}$. This completes the proof of our Theorem.

\section{REFERENCES}

1. Chao Ko, Acta Sci. Natur. Univ. Szechuan. 2 (1960), 57-64.

2. On the Diophantine equation $x^{2}=y^{n}+1, x y \neq 0$, Sci. Sinica 14 (1965), 457-460. MR 32 \# 1164.

3. T. Nagell, Sur l'impossibilité de l'equation indéterminée $y^{2}=z^{p}+1$, Norsk. Mat. Forenings Skrifter I 4 (1921).

Department of Mathematics, Pennsylvania State University, University Park, PennsylVANIA 16802 